\title{
Double-blind peer review
}

\author{
Nature and the Nature journals start offering anonymity to authors during the peer-review process.
}

Beginning in March 2015, Nature, Nature Chemical Biology and the other monthly Nature Research Journals will experiment with an alternative to their time-tested method of peer review. Instead of the traditional single-blind method, in which reviewers are anonymous but know the authors' identity, authors will be able to choose double-blind peer review, in which both authors and reviewers are unknown to each other.

Alternatives to the traditional singleblind peer-review process are often proposed. Chief among them are doubleblind and open peer review, two apparent opposites as in the latter both the authors and reviewers are known to each other. But the reasons cited in favor of these two alternatives are different. On one hand, proponents of open peer review see its transparency as a way to encourage more civil and thoughtful reviewer commentsalthough others are concerned that it promotes a less critical attitude. On the other hand, advocates of double-blind peer review suggest that it eliminates ad hominem biases, such as those based on gender, seniority, reputation and affiliation (J. Am. Soc. Inf. Sci. Technol. 64, 132-161, 2013). It remains a matter of debate how effectively either method can achieve these aspirations while maintaining the required level of scientific criticism.

Nature experimented with open peer review in 2006, but despite expressed interest at the time, the uptake from both authors and reviewers was low, and the open reviews were not technically substantive. Views about open peer review are still evolving, as several journals continue to experiment with variations on this practice. Opinions about double-blind review, however, are remarkably consistent.

In one of the largest studies on peer review-a 2009 international and crossdisciplinary survey of more than 4,000 researchers (J. Am. Soc. Inf. Sci. Technol. $64,132-161,2013)-76 \%$ of respondents indicated that double blind was an effective peer-review system. (By comparison, open and single-blind peer review were considered effective by $20 \%$ and $45 \%$ of respondents, respectively.) More recently, reader surveys conducted by Nature Publishing Group confirmed the desire to have double-blind peer review as an option at Nature journals. Importantly, this sentiment is widely echoed in conversations with young scientists worldwide. These conversations illustrate a widespread perception that biases based on authorship affect the traditional singleblind peer review and greatly influenced our decision to explore double-blind peer review.

Nature journals editors have historically not embraced double-blind peer review owing to skepticism of its efficacy, the potential difficulty of recruiting referees and an understanding that was an editorial responsibility to mitigate review bias. All editors take, and will continue to take, this responsibility seriously by maintaining awareness of any potential predispositions when selecting reviewers and considering their comments. They will also continue to honor reasonable requests to exclude particular reviewers, regardless of the chosen method of peer review. But by definition, unconscious biases may be difficult to identify and to control. Several studies have detected involuntary biases, notably on the basis of gender, in other areas of the scientific enterprise, such as the hiring of laboratory staff, citation habits and speaker lineups at conferences. Though it is difficult to guarantee a bias-free process, offering double-blind peer review as an option is a step in the right direction.

Since June 2013, Nature Geoscience and Nature Climate Change have allowed authors to choose between double-blind and single-blind peer review at submission. The uptake of the double-blind method has been much lower than the enthusiasm expressed in surveys would have predicted-no more than a fifth of monthly submissions are going the double-blind route-and, encouragingly, no substantial effects on the quality of reviews have been detected. However, the reactions to the trial among surveyed authors have been sufficiently positive that Nature and the Nature monthly journals have decided to join the experiment. (Nature Communications will join at a later date.)

The responsibility for rendering the manuscript anonymous falls to the authors. Clearly, in some situations, keeping the authors' identity secret will be impossible because of awareness of their work in the specialist community. We also continue to promote policies that support researchers who wish to release data early and to discuss their work with their peers prior to publication, via conferences or preprint servers. Therefore, the double-blind process is optional; some authors will choose it to assuage concerns about biases, others purely by principle.

We will keep this initiative under review, and we welcome comments from authors and reviewers. 